\title{
Immune Cell Landscape in Gastric Cancer
}

\author{
Yang Yang $\mathbb{D},{ }^{1,2,3}$ Wei He, ${ }^{1,2}$ Zi-rui Wang, ${ }^{1,2}$ Yu-jiao Wang, ${ }^{1,2}$ Lan-lan Li, ${ }^{1,4,5,6}$ \\ Jian-zhong Lu, ${ }^{1,4,5,6}$ Yan Tao, ${ }^{1,4,5,6}$ Jing Zhang, 1,4,5,6 Sheng-jun Fu, ${ }^{1,4,5,6}$ Zhi-ping Wang, ${ }^{1,4,5,6}$ \\ and Shan-hui Liu $\mathbb{D i D}^{1,4,5,6}$
}

\author{
${ }^{1}$ The Second Hospital of Lanzhou University, Lanzhou University, Lanzhou, 730000 Gansu, China \\ ${ }^{2}$ The Second Clinical Medical College of Lanzhou University, Lanzhou University, Lanzhou, 730000 Gansu, China \\ ${ }^{3}$ Department of Medical Oncology, Cancer Center, West China Hospital, Sichuan University, Chengdu, 610041 Sichuan, China \\ ${ }^{4}$ Institute of Urology, Lanzhou University Second Hospital, Lanzhou, 730000 Gansu, China \\ ${ }^{5}$ Key Laboratory of Urological Disease of Gansu Province, Lanzhou, 730000 Gansu, China \\ ${ }^{6}$ Institute of Gansu Nephro-Urological Clinical Central, Lanzhou, 730000 Gansu, China
}

Correspondence should be addressed to Shan-hui Liu; liushh2014@lzu.edu.cn

Received 15 August 2020; Revised 28 November 2020; Accepted 14 December 2020; Published 9 January 2021

Academic Editor: Lei Zhang

Copyright (c) 2021 Yang Yang et al. This is an open access article distributed under the Creative Commons Attribution License, which permits unrestricted use, distribution, and reproduction in any medium, provided the original work is properly cited.

Background. The tumor-infiltrating immune cells are closely associated with the prognosis of gastric cancer (GC). This article is aimed at determining the composition change of immune cells and immune regulatory factors in GC and normal tissues, depicting their prognosis value in GC, and revealing the relationship between them and GC clinical parameters. Methods. We used CIBERSORT to calculate the proportion of 22 immune cells in the GC or normal tissues; a $t$-test was applied to assess the expression difference of immune cells and immune regulatory factors in normal and GC tissues. The relationship of the immune cells, immune regulatory factors, and GC patients' clinical characteristics was assessed by univariate analysis. Results. In this study, we found that the proportion of macrophages increased, while plasma cells and monocytes decreased in GC tissues. In these immune fractions, Tregs and naïve B cells were found to be correlated with GC patients' prognosis. Interestingly, the expression of immune regulatory factors was ambiguous with their classical function in GC tissues. For example, TIM-3, FOXP3, and CMTM6 were overexpressed, while CD27 and PD-1 were underexpressed in GC tissues. We also found that IDO1, PD-1, TIGIT, and TIM-3 were highly expressed in high-grade GC tissues, the HERC2 expression level was related to patients' gender, and the TIGIT expression level was sensitive to targeted therapy. Furthermore, our results suggested that the infiltration of Tregs and naive B cells was strongly correlated with the $\mathrm{T}$ stage, radiation therapy, targeted molecular therapy, and the expression levels of TIM-3 and FOXP3 in GC. Conclusion. The expression pattern of tumor-infiltrating immune cells and immune regulatory factors was systematically depicted in the GC tumor microenvironment, indicating that individualized treatment based on the tumor-infiltrating immune cells and immune regulatory factors may be beneficial to GC patients.

\section{Introduction}

Gastric cancer (GC) is one of the most common gastrointestinal malignancies with high morbidity and mortality [1]. Although current traditional methods (such as surgery, chemotherapy, and radiotherapy) have a curative effect for GC patients, more than $50 \%$ of GC patients relapse from these treatments and GC further develop into metastatic gastric carcinoma [2,3]. Therefore, clarifying the tumorigenesis mechanism and finding a new treatment target and effective prognosis indexes are urgent. Immune infiltration plays an important role in GC tumorigenesis [4], and tumor immunotherapy has been paid more attention and has been used in clinical trials for GC $[5,6]$.

Cancer immunotherapy is aimed at improving the immune system's ability to eliminate cancer cells. Several types of immunotherapies have been developed to improve the immune system [7, 8]. Early immunotherapy is mainly focused on activating nonspecific immune cells such as NK cells and dendritic cells by cytokine stimulation [9]. 
However, these cells are poorly specific to tumor cells. So its clinical application was restricted. In recent years, with the further understanding of the character of T cells and B cells in the tumor environment, immunotherapy has been developed into activating the adaptive immune cells and it has shown a great advantage [10]. In particular, numerous research signs of progress have been achieved on chimeric antigen receptor $\mathrm{T}$ cells [11]. Besides, strategies for enhancing immune checkpoint blockade therapy, such as PD1/PD-L1/TIM-3 inhibitors and monoclonal antibodies, also showed great potential in tumor immunotherapy $[12,13]$.

Due to the highly complex composition of the tumor microenvironment, the immune cells and immune regulatory factors have not been systematically studied in GC tissues. The relationship between immune cells and clinical-pathological parameters and immune regulatory factors also need to be explored. The Cancer Genome Atlas (TCGA) program, a landmark cancer genomics program, was designed to molecularly characterize over 20,000 primary cancer and matched normal samples [14]. Benefiting from the bioinformatics tool, CIBERSORT, we could systematically analyze the tumor-infiltrating immune cells in the tumor environment [15].

In this study, we calculated the proportion of 22 types of tumor-infiltrating immune cells and evaluated the expression of immune regulatory factors in human GC tissues and normal tissues. The correlation between immune cells, clinicalpathological parameters, and immune regulatory factors was also assessed. By systematically analyzing the prognosis value of the tumor-infiltrating immune cells and immune regulatory factors in GC, we hope this work would be helpful for us to understand the GC tumor environment and would be useful for GC clinical immunotherapy.

\section{Results}

2.1. Overview of Clinical Data of 256 Patients. In this article, we have collected 256 patients' clinical and pathological information (Table 1).

2.2. Adaptive Immune Cells in Gastric Cancer. In normal and GC tissues, the fraction of naïve B cells slightly increased in GC although there was no significant difference. In GC, total B cells (95\% CI $-0.293--0.190 ; P<0.0001)$ and plasma cells (95\% CI $-0.282--0.220 ; \quad P<0.0001)$ were significantly decreased (Figure 1(a)).

Like naïve $\mathrm{B}$ cells, $\mathrm{CD} 4^{+} \mathrm{T}$ cells were slightly higher in GC than in normal tissues although there was no significant difference. $\mathrm{CD}^{+} \mathrm{T}$ cells and resting memory $\mathrm{CD} 4^{+}$ $\mathrm{T}$ cells decreased in GC tissues. In contrast, the fractions of Tregs and activated memory CD $4^{+} \mathrm{T}$ cells (95\% CI $0.011-$ $0.065 ; P=0.01$ ) were significantly higher in GC than in normal tissues, especially the activated memory $\mathrm{CD} 4^{+} \mathrm{T}$ cells (Figure 1(b)).

2.3. Innate Immune Cells in Gastric Cancer. In GC tissues, the innate immune cell fractions, such as total NK cells, resting NK cells, and activated NK cells, increased (Figure 2(a)). Besides, the fractions of total DC cells, resting DC cells, and
TABLE 1: Primary tumor characteristics.

\begin{tabular}{|c|c|c|c|}
\hline Variable & Number of samples & $\%$ & Valid (\%) \\
\hline \multicolumn{4}{|c|}{ Age at diagnosis } \\
\hline$\leq 50$ & 18 & 7.0 & 7.5 \\
\hline$>50$ & 223 & 87.1 & 92.5 \\
\hline Missing & 15 & 5.9 & \\
\hline \multicolumn{4}{|l|}{ Gender } \\
\hline Male & 93 & 36.3 & 38.0 \\
\hline Female & 152 & 59.4 & 62.0 \\
\hline Missing & 11 & 4.3 & \\
\hline \multicolumn{4}{|l|}{ Stage } \\
\hline I & 25 & 9.8 & 11.2 \\
\hline II & 77 & 30.1 & 34.5 \\
\hline III & 100 & 39.1 & 44.8 \\
\hline IV & 21 & 8.2 & 9.4 \\
\hline Missing & 33 & 12.9 & \\
\hline \multicolumn{4}{|l|}{ Grade } \\
\hline G1 & 6 & 2.3 & 2.6 \\
\hline G2 & 67 & 26.2 & 28.5 \\
\hline G3 & 162 & 63.2 & 68.9 \\
\hline Missing & 21 & 8.2 & \\
\hline \multicolumn{4}{|l|}{ T stage } \\
\hline $\mathrm{T} 1$ & 5 & 2.0 & 2.1 \\
\hline $\mathrm{T} 2$ & 55 & 21.5 & 23.3 \\
\hline T3 & 110 & 43.0 & 46.6 \\
\hline $\mathrm{T} 4$ & 66 & 25.8 & 28.0 \\
\hline Missing & 20 & 7.8 & \\
\hline \multicolumn{4}{|c|}{ Radiation treatment } \\
\hline True & 23 & 9.0 & 20.9 \\
\hline False & 87 & 34.0 & 79.1 \\
\hline Missing & 20 & 57.0 & \\
\hline \multicolumn{4}{|c|}{ Targeted molecular therapy } \\
\hline True & 51 & 19.9 & 45.9 \\
\hline False & 60 & 23.4 & 54.1 \\
\hline Missing & 145 & 56.6 & \\
\hline
\end{tabular}

activated DC cells were also overexpressed in GC tissues (Figure 2(b)). The expression fraction of activated mast cells was higher in GC tissues although the slight fraction change showed no significant difference (Figure 2(c)). There was no significant change of the eosinophil and neutrophil fraction. Monocytes (95\% CI $-0.012--0.001 ; P=0.02$ ) were significantly reduced in GC (Figure $2(\mathrm{~d})$ ). Total macrophage fraction (95\% CI $0.145-0.272 ; P<0.0001)$ was significantly highly expressed in GC tissues. The fractions of M0 $(95 \%$ CI $0.050-0.163 ; P=0.0003)$, M1 (95\% CI 0.027-0.070; $P<$ 0.0001 ), and M2 macrophages (95\% CI 0.021-0.087; $P=$ 0.0014 ) were higher in GC tissues. M2/M1 macrophages in GC tissues slightly decreased, but there was no significant change (Figures 2(e) and 2(f)).

2.4. Correlation with 22 Immune Cells in Gastric Cancer. Based on the fraction proportion of the tumor-infiltrating 

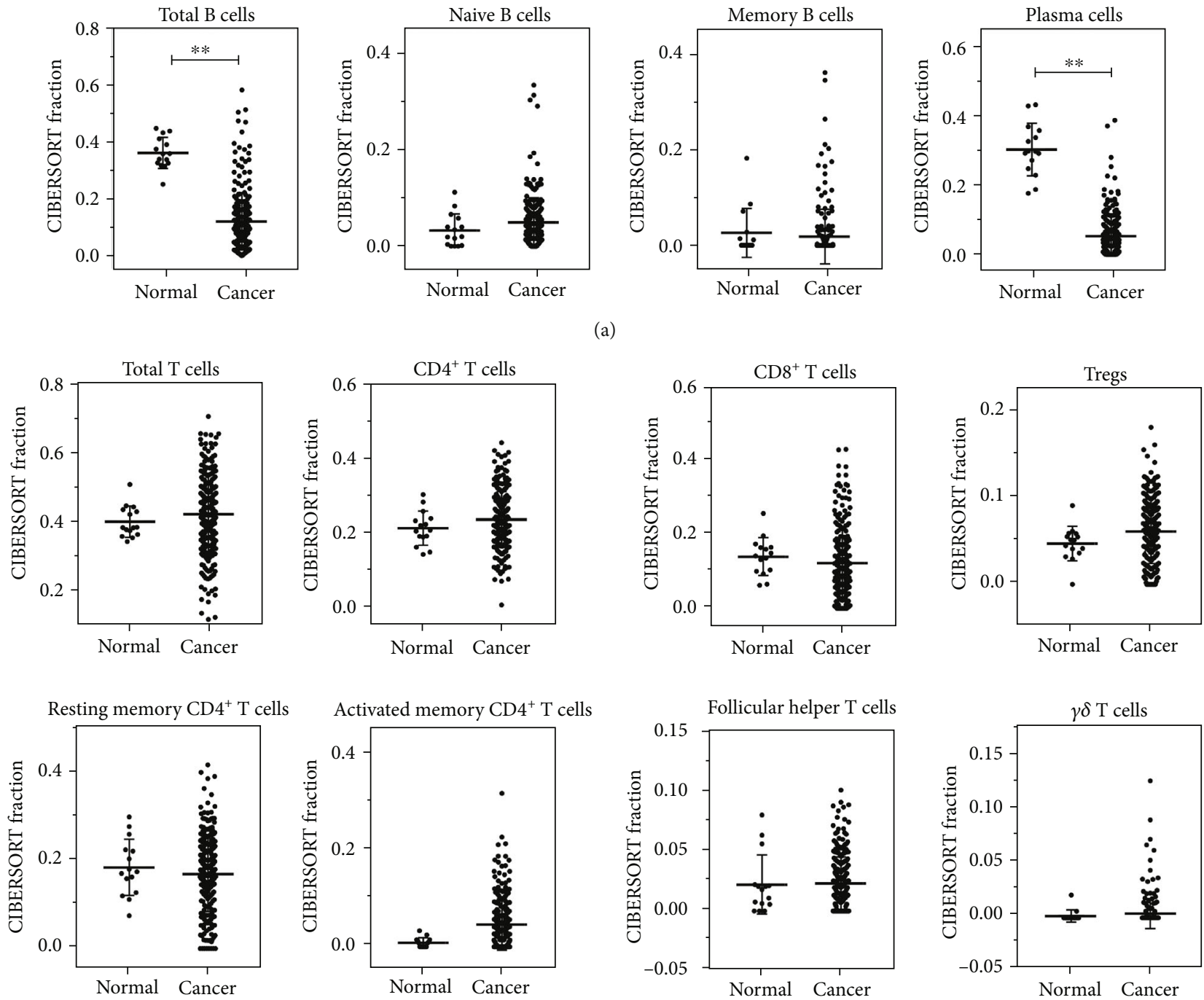

(b)

FIgure 1: Adaptive immune cells in human GC tissue and healthy gastric tissue. (a) Total B cells, naïve B cells, memory B cells, and plasma cells and (b) total $\mathrm{T}$ cells, $\mathrm{CD} 4^{+} \mathrm{T}$ cells, $\mathrm{CD} 8^{+} \mathrm{T}$ cells, Tregs, resting memory $\mathrm{CD} 4^{+} \mathrm{T}$ cells, activated memory $\mathrm{CD} 4^{+} \mathrm{T}$ cells, follicular helper $\mathrm{T}$ cells, and $\gamma \delta$ T cells were calculated for each patient group and compared using $t$-test analysis. ${ }^{*} P<0.05 ;{ }^{* *} P<0.01$.

immune cells, we calculated the correlation between the fractions of 22 immune cells with the corrplot $\mathrm{R}$ package (Figure 3). Results showed that a positive correlation existed between activated mast cells and neutrophils. The activated memory $\mathrm{CD} 4^{+} \mathrm{T}$ cells showed a strong positive correlation with $\mathrm{CD} 8^{+} \mathrm{T}$ cells and $\mathrm{M} 1$ macrophages. The fraction of resting memory $\mathrm{CD} 4^{+} \mathrm{T}$ cells negatively correlated with activated memory $\mathrm{CD}^{+} \mathrm{T}$ cells and $\mathrm{CD} 8^{+} \mathrm{T}$ cells. A negative correlation also existed in $\mathrm{CD}^{+} \mathrm{T}$ cells and $\mathrm{M} 0$ macrophages.

2.5. Prognostic Evaluation of Tumor-Infiltrating Immune Cells. Based on the expression of tumor-infiltrating immune cells, we divided these GC patients into the high-infiltrating immune group and low-infiltrating immune group. KaplanMeier survival analysis was performed to analyze the tumor-infiltrating immune cell prognosis value. Results showed that patients with low-infiltrating naïve B cells
(Figure 4(a)) and high-infiltrating Tregs (Figure 4(b)) had a better prognosis.

2.6. Immune Regulatory Factor Expression in GC Tissues and Normal Tissues. Considering that immune regulatory factors play a vital role to modulate the tumor-infiltrating immune cell function, we investigated the expression of many important immune regulatory factors in GC tissues and normal tissues. As shown in Figure 5(a), the expression levels of TIM-3 (95\% CI 1.341-4.781; $P=0.0005)$, FOXP3 (95\% CI 0.384$2.948 ; \quad P=0.0111)$, CMTM6 (95\% CI 2.80-14.94; $P=$ $0.0044)$, CTSB (95\% CI 18.51-213.5; $P=0.0199)$, HERC2 (95\% CI $0.563-2.183 ; P=0.0010)$, MTOR (95\% CI $1.131-$ 4.35; $P=0.0009)$, CD47 (95\% CI 4.255-12.97; $P=0.0001)$, and CD276 (95\% CI 5.941-14.36; $P<0.0001)$ were significantly higher in GC tissues than in normal gastric tissues. In contrast, CD27 (95\% CI -30.11--7.72; $P=0.0010)$ showed 


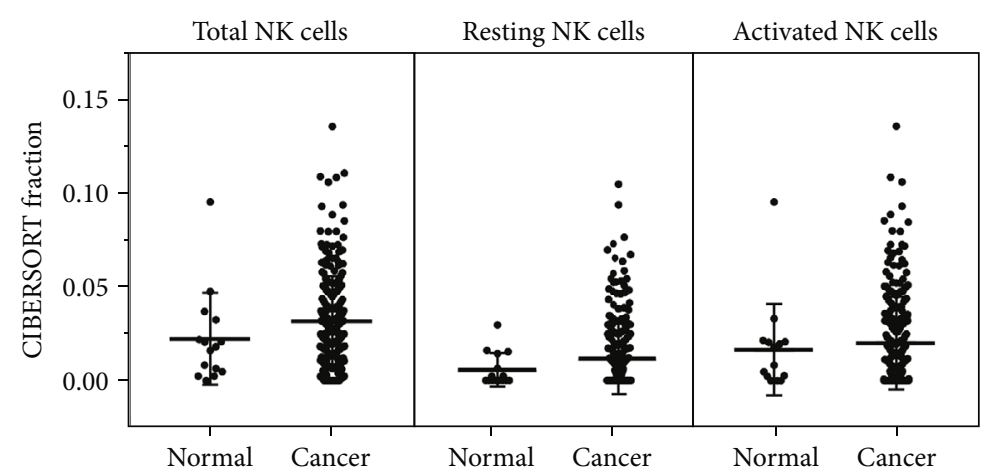

(a)

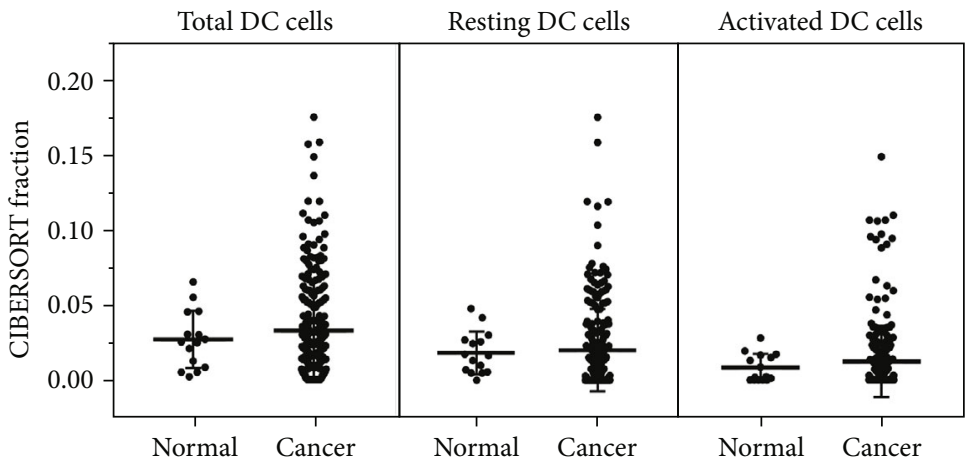

(b)

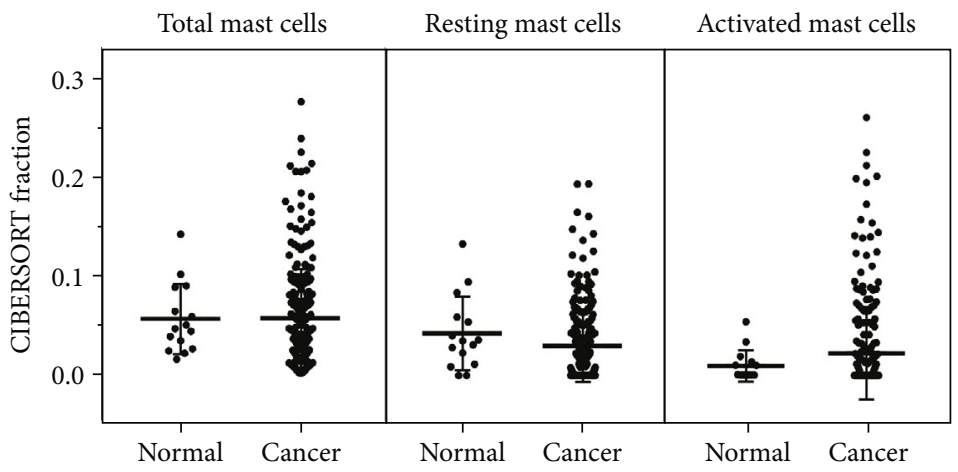

(c)

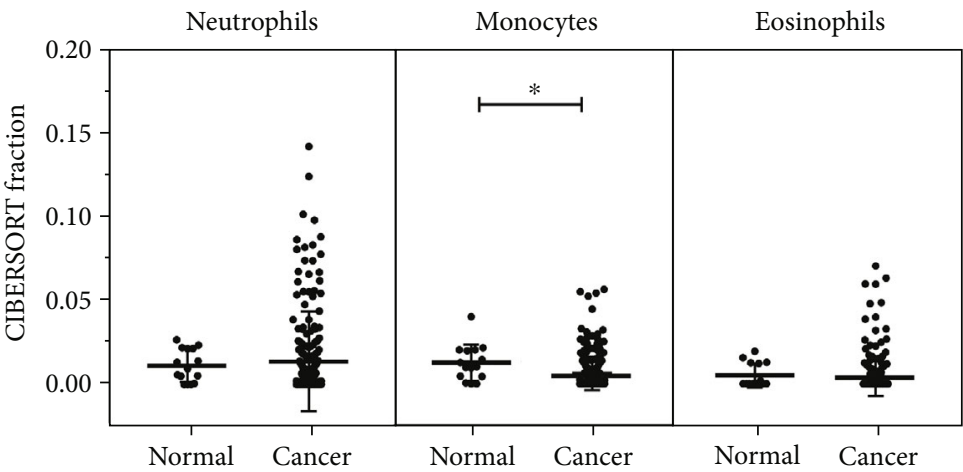

(d)

Figure 2: Continued. 


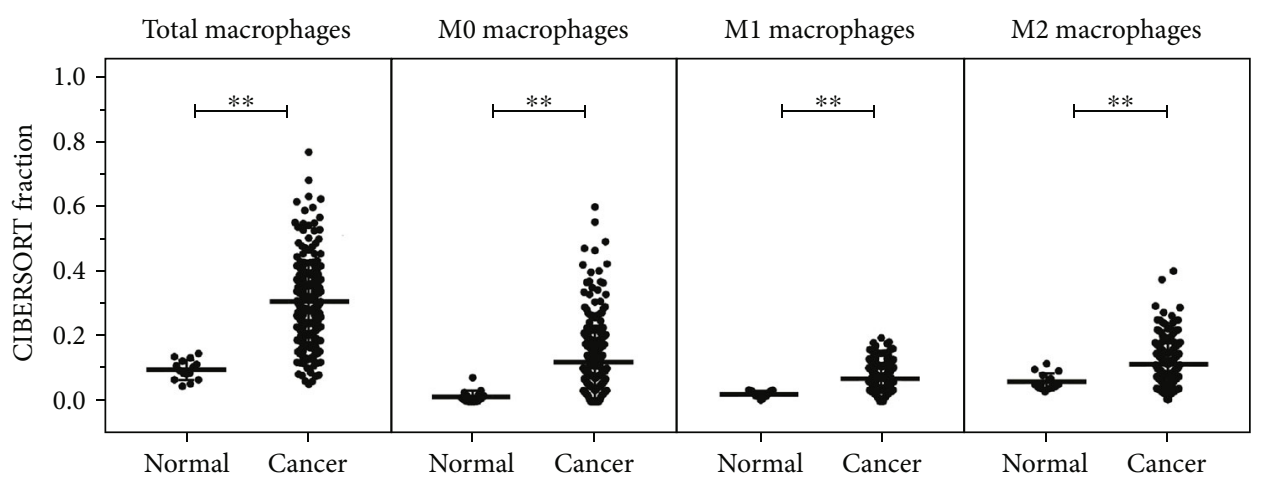

(e)

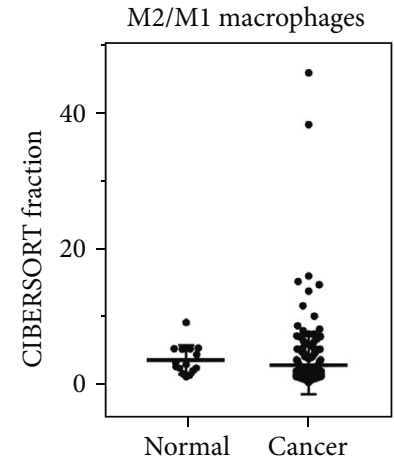

(f)

FIgURe 2: Innate immune cells in human GC tissue and healthy gastric tissue. Total nonspecific immune response cells in human GC and normal gastric tissues. (a) Total NK cells, resting NK cells, and activated NK cells; (b) total dendritic cells, resting dendritic cells, and activated dendritic cells; (c) total mast cells, resting mast cells, and activated mast cells; (d) neutrophils, monocytes, and eosinophils; (e) total macrophages, M0 macrophages, M1 macrophages, and M2 macrophages; and (f) M2/M1 macrophages were calculated for each patient group and compared using $t$-test analysis. ${ }^{*} P<0.05 ;{ }^{* *} P<0.01$.

high expression in normal tissues. In addition, as shown in Figure 5(b), we found that the expression levels of CTLA4, TIGIT, IDO1, PD-L1, and PD-L2 were higher in GC, and PD-1 expression was slightly lower in GC tissues.

2.7. Correlation between Tumor-Infiltrating Immune Cells, Immune Regulatory Factors, and Clinical Pathological Parameters. The relationship between age, gender, clinical stage, grade, $\mathrm{T}$ stage, radiotherapy, and targeted molecular therapy and fractions of naïve B cells or Tregs was assessed by univariate analysis. Results suggested that naïve B cells $(\mathrm{HR}=0.443$, 95\% CI 0.205-0.957, $P=0.038$; $\mathrm{HR}=0.542$, 95\% CI $0.296-0.991, P=0.047)$ and Tregs $(\mathrm{HR}=0.384$, 95\% CI 0.191-0.772, $P=0.007$; HR $=0.575,95 \%$ CI $0.333-$ 0.994, $P=0.047$ ) were less infiltrating in the GC patients who received radiation therapy and targeted molecular therapy (Figure 6(a)).

The relationship between clinical-pathological parameters and immune regulatory factors was assessed by $t$-test analysis. Result showed that the gene expression levels of TIM-3 (95\% CI 0.298-2.277; $P=0.0113$ ), CD47 (95\% CI 0.510-7.738; $P=0.0258)$, IDO1 (95\% CI 0.450-68.660; $P=$ $0.0468)$, PD-1 (95\% CI 0.180-2.507; $P=0.0241)$, and TIGIT (95\% CI 0.144-1.171; $P=0.0126)$ increased in the high-grade group. CMTM6 (95\% CI 0.379-14.810; $P=0.0393$ ) also showed high expression in GC patients bearing a high stage. HERC2 (95\% CI 0.061-1.444; $P=0.0332$ ) expression was significantly higher in male GC patients, whereas TIGIT (95\% CI -0.980--0.028; $P=0.0383$ ) decreased in GC patients who received targeted therapy (Figure 6(b)).

Univariate analysis was applied to analyze the relationship between the expression of TIM-3, FOXP3, CMTM6, CTSB, HERC2, MTOR, CD27, CD47, and CD276 and naïve $\mathrm{B}$ cells or Tregs. The results showed that infiltration of naive $B$ cells $(\mathrm{HR}=2.060,95 \%$ CI $1.276-3.325, P=0.003)$ and Tregs $(\mathrm{HR}=1.600,95 \%$ CI 1.096-2.335, $P=0.015)$ increased in GC tumor tissues with high expression of TIM-3. In the GC tissues with high HERC2 expression, infiltration of naïve
B cells $(\mathrm{HR}=0.646,95 \%$ CI $0.426-0.980, P=0.040)$ was decreased (Figure 6(c)).

\section{Discussion}

Gastric carcinoma, a highly common malignant tumor, is mainly treated by surgery, chemotherapy, and radiotherapy. However, these treatment options always do not work for some GC patients [16]. Nowadays, targeted therapy and immunotherapy showed more attractive attention for their specific targeting ability and low toxicity, especially to advanced gastric cancer [16-18]. The GC tumor environment is highly complex and heterogeneous. Further understanding of each composition proportion and interrelationship will be helpful for GC therapy. Benefiting from TCGA database and the bioinformatics tool, CIBERSORT, we could systematically investigate the tumor-infiltrating immune cells and the expression of many key immune regulatory factors in GC tissues. This makes it possible to explore their relationship to clinical-pathological parameters and their potential prognosis value.

Herein, we investigated the fraction of tumor-infiltrating immune cells by dividing them into cellular immunity and humoral immunity components. We found that the number of $\mathrm{CD}^{+} \mathrm{T}$ cell fraction increased in $\mathrm{GC}$, and $\mathrm{CD}^{+} \mathrm{T}$ cell fraction decreased. The tumor antigen-specific $\mathrm{CD} 8^{+} \mathrm{T}$ cells are negatively regulated by PD-1 and TIM-3 in GC [19]. Therefore, we believed that targeted therapy by increasing the level of $\mathrm{CD}^{+} \mathrm{T}$ cells would be an effective method to inhibit GC. Tregs could promote tumorigenesis and tumor immune escape by secreting immunosuppressive cytokines such as TGF- $\beta$ which can promote the expression of antiapoptotic molecules and help tumor cells to defend against apoptosis [20, 21]. Interestingly, in our work, high Treg infiltration portended a more favorable prognosis in GC. This result was consistent with other group studies where the high expression of FOXP3, a specific transcriptional regulator in Tregs, also showed a favorable prognosis [22-24]. In 


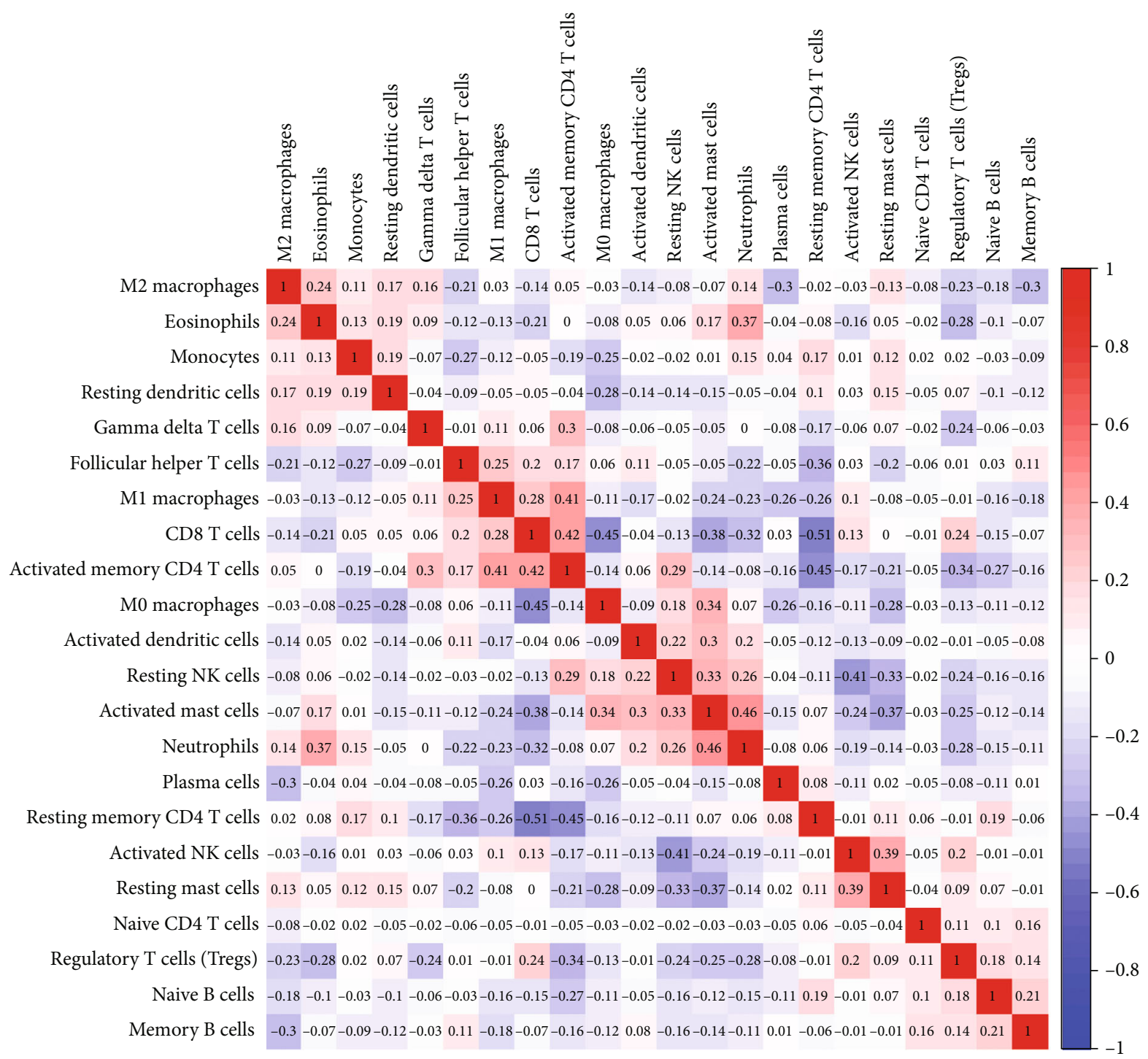

FIGURE 3: The relationship between 22 immune cells in GC. The red squares represent a positive correlation between the two immune cells, and the blue squares represent a negative correlation between the two immune cells. The numerical size represents the correlation. The larger the number, the stronger the correlation.

addition, patients who received radiation therapy and targeted molecular therapy always often were accompanied by low Treg infiltration. Since low Treg infiltration showed a poor prognosis, improving the radiation therapy and targeted molecular therapy may be urgently needed in GC.

Tumor-associated macrophages (TAM) are the largest fraction of tumor-infiltrating immune cells in the tumor environment [25]. They could be characterized by M0 macrophages, M1 macrophages, and M2 macrophages [26]. It was always believed that M1 macrophages possessed the ability to eliminate tumor cells. In contrast to M1 macrophages, M2 macrophages could express a variety of immunosuppressive factors and chemokines, which inhibit antitumor immunity by reducing antigen presentation and inhibiting $\mathrm{T}$ cell function [27]. Compared to the normal tissues, the fraction proportion of M1 and M2 macrophages increased significantly in GC. M2/M1 proportion decreased slightly with no significant change [28]. Therefore, based on the different immune effects of M1 and M2 in the tumor environment, we assumed that the strategy to induce the M1 macrophage expression and inhibit the M2 macrophage expression may be also useful to improve the therapeutic effect for GC patients.

In the tumor microenvironment of gastric cancer, it was found that when mast cells were activated, they could produce and secrete neutrophil chemotactic factors, which in turn promotes an increase in the recruitment of neutrophils [29]. Other studies have shown that mast cells secrete lymphangiogenic factors to promote the formation of lymphatic vessels, which in turn promotes the production of neutrophils [30]. We found that activated mast cells were positively correlated with neutrophils. These studies are consistent with our findings.

Immune regulatory factors play a key role in tumorigenesis and in immunotherapy directly or indirectly. For example, FOXP3 is always highly expressed in the tumor environment 


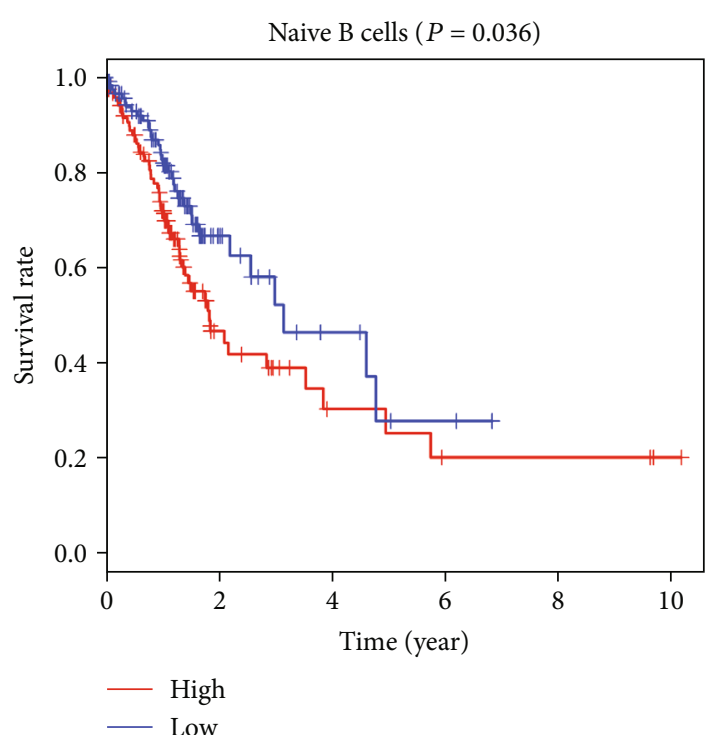

(a)

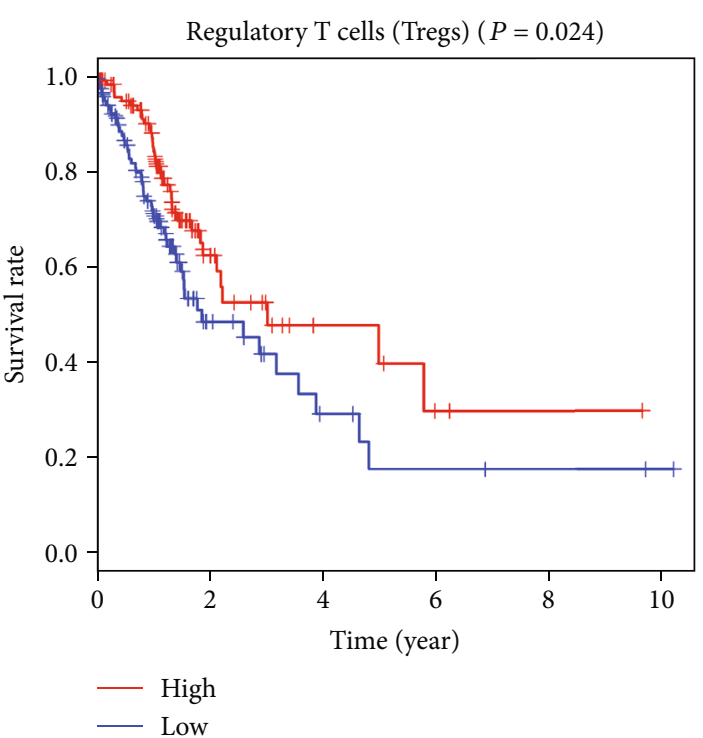

(b)

FIGURE 4: Kaplan-Meier curves and overall survival of high-infiltrating (a) naïve B cells and (b) Tregs in GC patients.

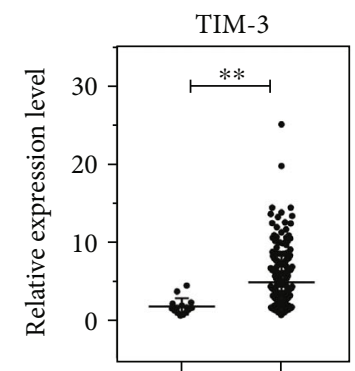

Normal Cancer
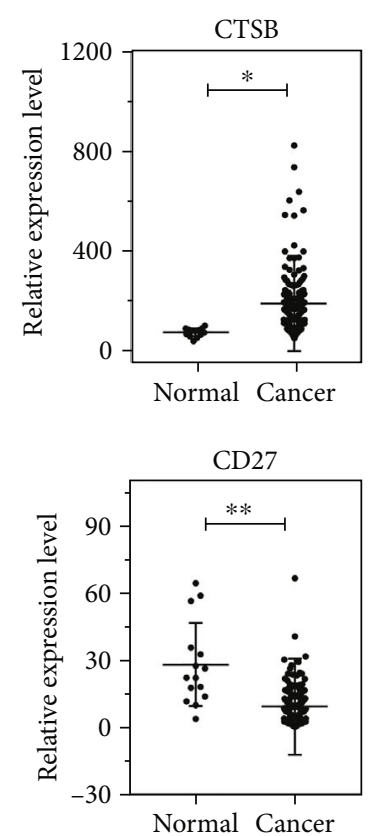
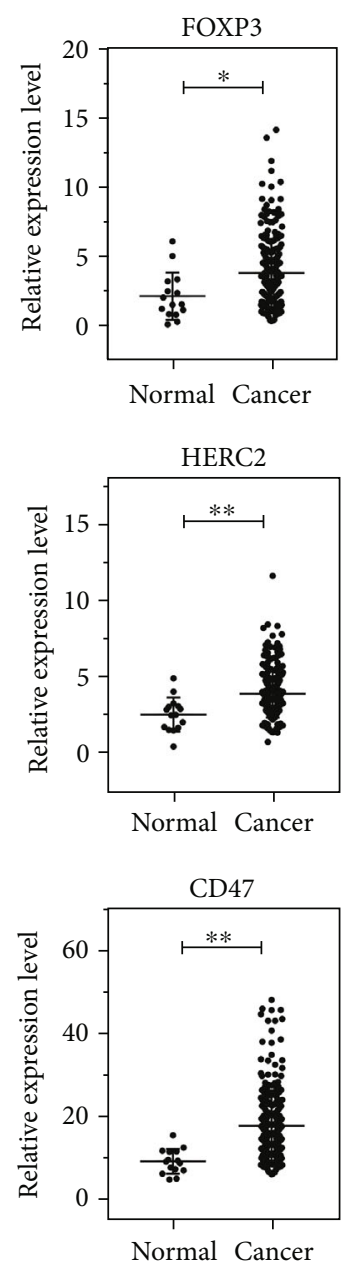

(a)
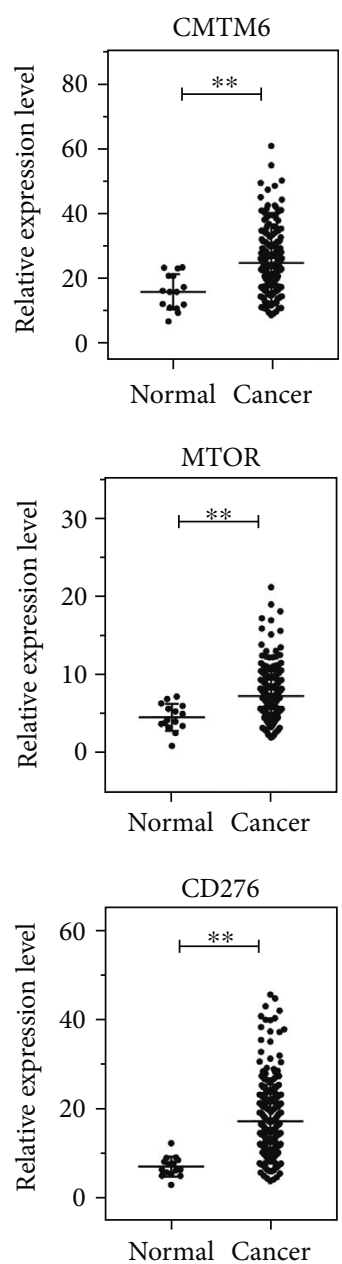

FIgURE 5: Continued. 

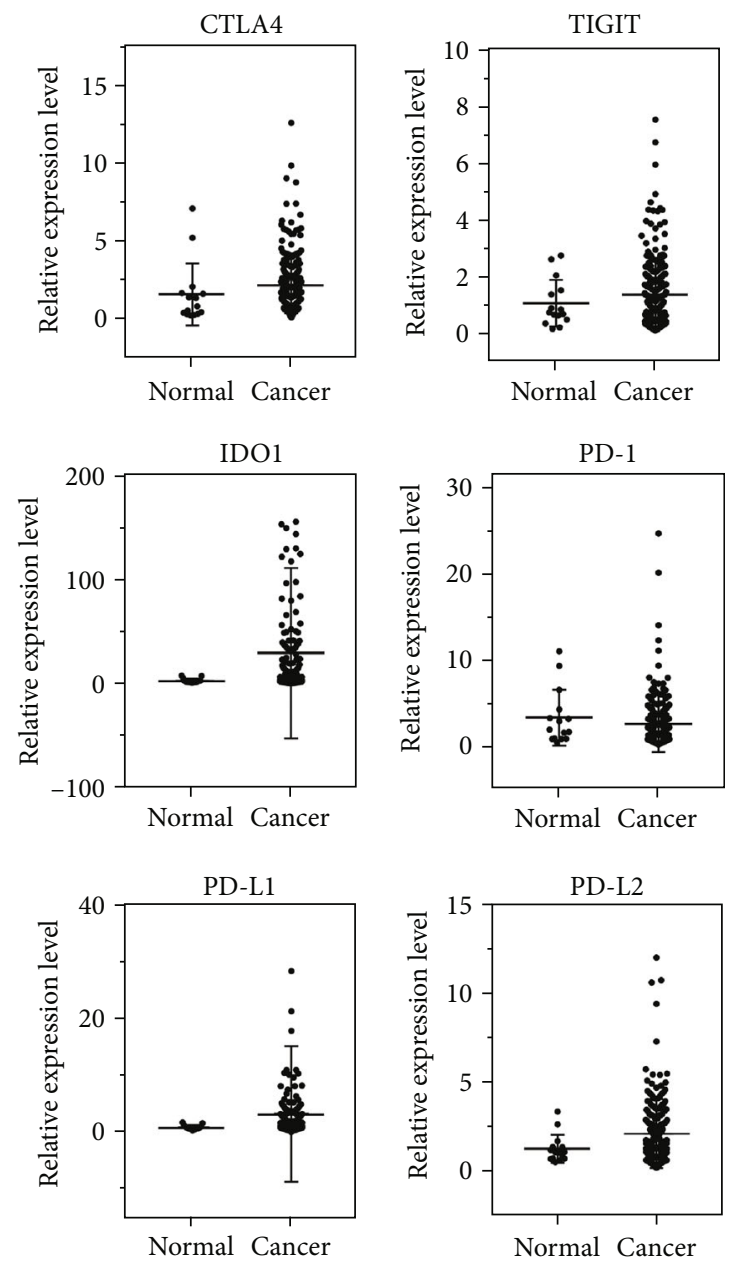

(b)

FIGURE 5: Immune regulatory factor expression in human GC and normal gastric tissues: (a) TIM-3, FOXP3, CMTM6, CTSB, HERC2, MTOR, CD27, CD47, and CD276; (b) CTLA4, TIGIT, IDO1, PD-1, PD-L1, and PD-L2. ${ }^{*} P<0.05$; ${ }^{*} P<0.01$.

and is capable of mediating immune escape [31, 32]. High expression of FOXP3 was correlated with a poor prognosis [33]. Our study also showed the high expression of FOXP3 in GCs, and the expression of FOXP3 is associated with the fraction of naïve B cells and Tregs in GCs. CD27/CD70 signaling promotes effector and memory $\mathrm{T}$ cell differentiation and enhances B cell and NK cell activation and function [34]. CD27/CD70 is expressed in CD20 $0^{+} \mathrm{B}$ cells and $\mathrm{CD} 8^{+} \mathrm{T}$ cells in the tumor microenvironment of gastric cancer. These cells are involved in antitumor immunity and are associated with survival in gastric cancer patients $[35,36]$.

In this work, we have identified and explored the expression of several key immune regulatory factors and their relationship to tumor-infiltrating immune cells, GC patients' tumor grade, tumor stage, and some other clinical parameters. Our work indicated that CD27 was underexpressed in GC. Furthermore, the expression of $\mathrm{CD}^{+} \mathrm{T}$ cells also decreased in GC tissues. As the interaction of CD27 on a T cell and CD70 on a B cell enhances $\mathrm{T}$ cell activation in terms of proliferation, we speculated that inducing CD27 expression may be a promising way to activate $\mathrm{CD} 8^{+} \mathrm{T}$ cells in GCs. TIM-3, CTLA4, and TIGIT primarily trigger peripheral tolerance and promote tumor growth by inhibiting the activity of effector T cells $[37,38]$. Highly expressed TIM-3 is closely related to the poor prognosis of gastric cancer [39]. CTLA4 is an important immune checkpoint for tumor immunotherapy, such as the CTLA4 inhibitor ipilimumab, which has been tested in clinical trials in multiple types of tumors [40]. The high expression of TIGIT inhibits the antitumor function of CD8 T cells in the microenvironment of gastric cancer [41]. In our study, we did not observe a significant expression change of these genes. CD47 produces cascades that inhibit the phagocytosis of macrophages [42]. Here, we can observe that it was highly expressed in GC tissues. CD47 and TIM-3 expression levels in high-grade GC tissue were significantly increased. Interestingly, regarding the relationship between TIM-3 and GC prognosis, we found that high expression of TIM-3 shows a favorable prognosis. This result is consistent with Holderried et al.'s research [43]. Besides, we found that TIGIT expression decreases in patients who received targeted therapy.

PD-1 and PD-L1 restrict T lymphocyte antitumor function by inhibiting $\mathrm{T}$ cell activation [44]. Nowadays, PD1/PD-L1 inhibitors and antibodies have been applied to 


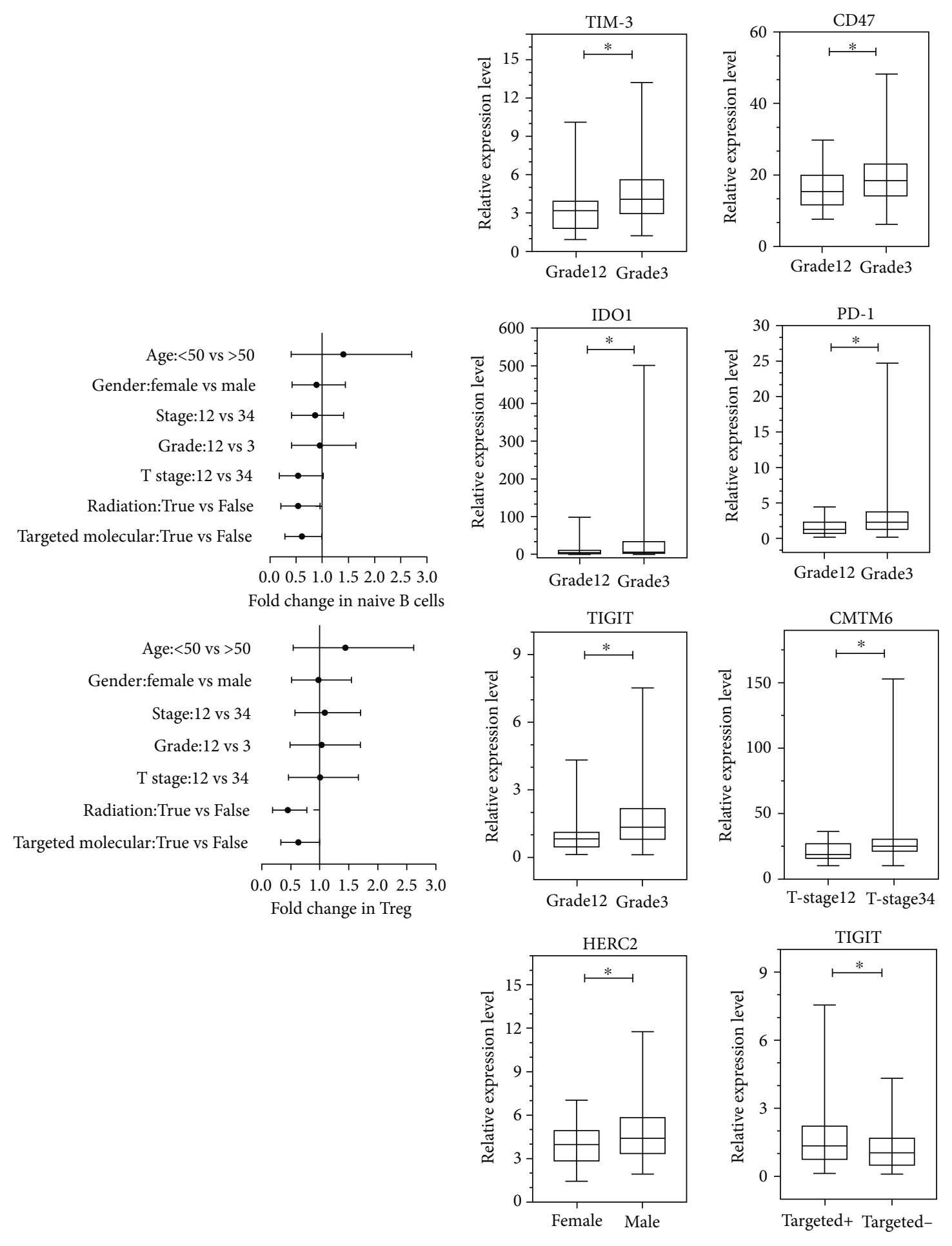

(a)

(b)

Figure 6: Continued. 

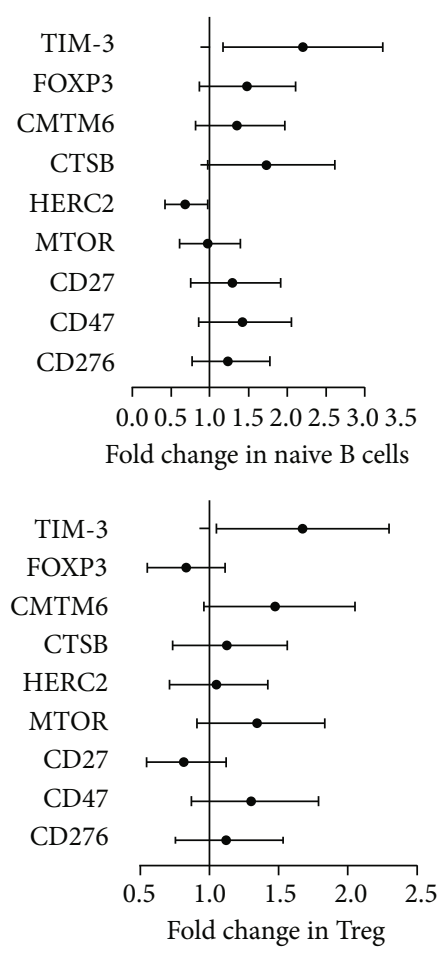

(c)

FIGURE 6: Relationship between clinical-pathological parameters and immune regulatory factors (a) and immune regulatory factors (b) in GC patients. Correlation between immune cells and immune regulatory factors (c) in GC. ${ }^{*} P<0.05 ;{ }^{* *} P<0.01$.

clinical trials. In this work, we did not observe a significant difference change of PD-1/PD-L1 expression in GC tissues and in normal tissues. Recently, research suggested that CMTM6 could block the degradation of PD-L1 and stabilize PD-L1 [45]. Our results showed that CMTM6 increased significantly in GC and was also upregulated in GC tissues with a high stage. Therefore, we assumed that CMTM6 may be a potential target for GC immunotherapy. HERC2 has been rarely studied, and our results showed that its expression is related to gender, which is significantly higher in men. This result was supported by the epidemiology result that the incidence of GC is twofold in males than in females [46]. Therefore, HEGC2 could be regarded as a result affected by gender. It may be a reference factor for individualized or personalized GC therapy. However, due to the limited sample size of the dataset, these studies still need more samples and research evidence to validate.

Taken together, our study is the first to identify the tumor-infiltrating immune cells and immune regulatory factors with their prognosis value and their correlation with the tumor clinical index. This study indicates that tumorinfiltrating immune cells are important determinants of prognosis in GC. Meanwhile, it reveals several potential targets and biomarkers for immunotherapy development.

\section{Materials and Methods}

4.1. Data Collection. All data in this study were downloaded from the public database. The normal gastric tissue $(N=32$ ) and GC tissue $(N=381)$ gene expression data and clinical information data were downloaded from The Cancer Genome Atlas (TCGA, https://cancergenome.nih.gov/). The corresponding clinical data, including age, gender, grade and stage, $\mathrm{T}$ stage, radiation therapy, targeted molecular therapy, and survival status, were also collected.

4.2. Immune Cell Evaluation. CIBERSORT is an analytical tool used to provide an estimation of the immune cell infiltration in a mixed cell population from their gene expression profile [47]. In this study, we used CIBERSORT to calculate the fraction of 22 infiltrating immune cells in normal gastric and GC tissues with the RNA expression data. These immune cell components included naïve $\mathrm{B}$ cells, memory $\mathrm{B}$ cells, naïve $\mathrm{CD} 4^{+} \mathrm{T}$ cells, resting memory $\mathrm{CD} 4^{+} \mathrm{T}$ cells, activated memory $\mathrm{CD}^{+}{ }^{+} \mathrm{T}$ cells, $\mathrm{CD}^{+} \mathrm{T}$ cells, follicular helper $\mathrm{T}$ cells, regulatory $\mathrm{T}$ cells (Tregs), gamma delta $(\gamma \delta) \mathrm{T}$ cells, resting dendritic (DC) cells, activated DC cells, M2 macrophages, M1 macrophages, M0 macrophages, resting natural killer (NK) cells, activated NK cells, resting mast cells, activated mast cells, plasma cells, monocytes, eosinophils, and neutrophils. The total B cells included naïve B cells, memory B cells, and plasma cells. The total $\mathrm{T}$ cells included naïve $\mathrm{CD} 4^{+} \mathrm{T}$ cells, resting memory $\mathrm{CD}^{+} \mathrm{T}$ cells, activated memory $\mathrm{CD}^{+} \mathrm{T}$ cells, $\mathrm{CD}^{+} \mathrm{T}$ cells, follicular helper T cells, Tregs, and $\gamma \delta \mathrm{T}$ cells.

4.3. Data Statistics. In this work, data analysis and statistics are performed by using $\mathrm{R}$. The survival time is defined from the diagnosed date to the dead time. Univariate analysis was used to assess the relationship between immune cells and clinical-pathological parameters and immune regulatory 
factors. A $t$-test was applied to assess the different expression levels of tumor-infiltrating immune cells and immune regulatory factors in normal and GC tissues. It was also used to analyze the clinical-pathological parameters and immune regulatory factors. $P$ value $<0.05$ was considered statistically significant $\left({ }^{*} P<0.05,{ }^{* *} P<0.01\right)$.

\section{Abbreviations}

$\begin{array}{ll}\text { GC: } & \text { Gastric cancer } \\ \text { NK cells: } & \text { Natural killer cells } \\ \text { TCGA: } & \text { The Cancer Genome Atlas } \\ \text { Tregs: } & \text { Regulatory T cells } \\ \gamma \delta \text { T cells: } & \text { Gamma delta T cells } \\ \text { DC cells: } & \text { Dendritic cells } \\ \text { TAM: } & \text { Tumor-associated macrophages. }\end{array}$

\section{Data Availability}

Our raw data types are RNA-seq, survival data, and clinical data. All data are downloaded from the public database (TCGA database: https://cancergenome.nih.gov/). And our data does not involve animal experiments, human clinical trials, etc., so ethical approval and other content are not required.

\section{Conflicts of Interest}

The authors declare that there are no conflicts of interest.

\section{Authors' Contributions}

Yang Yang and Wei He contributed to this work equally and should be regarded as co-first authors.

\section{Acknowledgments}

This work was supported by the Cuiying Scientific and Technological Innovation Program of Lanzhou University Second Hospital (Grant no. CY2018-QN13).

\section{Supplementary Materials}

Table S1: relationship between immune cell fraction and prognosis. Table S2: relationship between immune regulatory factor fraction and prognosis. Table S3: relationship between immune regulatory factors and clinical-pathological parameters. Figure S1: analysis of the prognosis value of tumorinfiltrating immune cells. Figure S2: analysis of the prognosis value of tumor-infiltrating immune cells. Figure S3: analysis of the prognosis value of immune regulatory factors. Figure S4: analysis of the prognosis value of immune regulatory factors. Figure S5: relationship between immune regulatory factors and clinical-pathological parameters. Figure S6: relationship between immune regulatory factors and clinicalpathological parameters. Figure S7: relationship between immune regulatory factors and clinical-pathological parameters. Figure S8: relationship between immune regulatory factors and clinical-pathological parameters. (Supplementary Materials)

\section{References}

[1] T. Ishii, A. Kawazoe, and K. Shitara, "Dawn of precision medicine on gastric cancer," International Journal of Clinical Oncology, vol. 24, no. 7, pp. 779-788, 2019.

[2] G. Jomrich and S. F. Schoppmann, "Targeted therapy in gastric cancer,” European Surgery, vol. 48, no. 5, pp. 278-284, 2016.

[3] Y. Chen, Q. Zhou, H. Wang et al., "Predicting peritoneal dissemination of gastric cancer in the era of precision medicine: molecular characterization and biomarkers," Cancers, vol. 12, no. 8, article 2236, 2020.

[4] R. Bai, N. Chen, T. Liang et al., "Novel frontiers of treatment for advanced gastric or gastroesophageal junction cancer (GC/GEJC): will immunotherapy be a future direction?," Frontiers in Oncology, vol. 10, p. 912, 2020.

[5] Y. Du and Y. Wei, "Therapeutic potential of natural killer cells in gastric cancer," Frontiers in Immunology, vol. 9, article 3095, 2019.

[6] T. Misumi, K. Tanabe, N. Fujikuni, and H. Ohdan, "Stimulation of natural killer cells with rhCD137 ligand enhances tumor-targeting antibody efficacy in gastric cancer," PLoS One, vol. 13, no. 10, article e0204880, 2018.

[7] F. M. Würfel, C. Winterhalter, P. Trenkwalder, R. M. Wirtz, and W. Würfel, "European patent in immunoncology: from immunological principles of implantation to cancer treatment," International Journal of Molecular Sciences, vol. 20, no. 8, article 1830, 2019.

[8] P. Gotwals, S. Cameron, D. Cipolletta et al., "Prospects for combining targeted and conventional cancer therapy with immunotherapy," Nature Reviews Cancer, vol. 17, no. 5, pp. 286-301, 2017.

[9] N. G. Minutolo, E. E. Hollander, and D. J. Powell Jr., "The emergence of universal immune receptor T cell therapy for cancer," Frontiers in Oncology, vol. 9, p. 176, 2019.

[10] V. Vijayan, S. Uthaman, and I. K. Park, "Cell membranecamouflaged nanoparticles: a promising biomimetic strategy for cancer theragnostics," Polymers, vol. 10, no. 9, p. 983, 2018.

[11] M. P. Brown, L. M. Ebert, and T. Gargett, "Clinical chimeric antigen receptor- $\mathrm{T}$ cell therapy: a new and promising treatment modality for glioblastoma," Clinical \& Translational Immunology, vol. 8, no. 5, article e1050, 2019.

[12] S. Kumar, R. V. Saini, and N. Mahindroo, "Recent advances in cancer immunology and immunology-based anticancer therapies," Biomedicine \& Pharmacotherapy, vol. 96, pp. 14911500, 2017.

[13] Y. He, J. Cao, C. Zhao, X. Li, C. Zhou, and F. R. Hirsch, “TIM3 , a promising target for cancer immunotherapy," Oncotargets and Therapy, vol. 11, pp. 7005-7009, 2018.

[14] Z. Wang, M. A. Jensen, and J. C. Zenklusen, "A practical guide to The Cancer Genome Atlas (TCGA)," Methods in Molecular Biology, vol. 1418, pp. 111-141, 2016.

[15] B. Chen, M. S. Khodadoust, C. L. Liu, A. M. Newman, and A. A. Alizadeh, "Profiling tumor infiltrating immune cells with CIBERSORT," Methods in Molecular Biology, vol. 1711, pp. 243-259, 2018.

[16] D. Zhao, S. J. Klempner, and J. Chao, "Progress and challenges in HER2-positive gastroesophageal adenocarcinoma," Journal of Hematology \& Oncology, vol. 12, no. 1, p. 50, 2019.

[17] X. Li, D. Tang, Y. Yao, W. Qi, and J. Liang, "Clinical significance and positive correlation of FoxM1 and Her-2 expression 
in gastric cancer," Clinical and Experimental Medicine, vol. 14, no. 4, pp. 447-455, 2014.

[18] C. Coutzac, S. Pernot, N. Chaput, and A. Zaanan, "Immunotherapy in advanced gastric cancer, is it the future?," Critical Reviews in Oncology/Hematology, vol. 133, pp. 2532, 2019.

[19] X. Lu, L. Yang, D. Yao et al., "Tumor antigen-specific $\mathrm{CD}^{+} \mathrm{T}$ cells are negatively regulated by PD-1 and Tim-3 in human gastric cancer," Cellular Immunology, vol. 313, pp. 43-51, 2017.

[20] R. Houot, L. M. Schultz, A. Marabelle, and H. Kohrt, "T-cellbased immunotherapy: adoptive cell transfer and checkpoint inhibition," Cancer Immunology Research, vol. 3, no. 10, pp. 1115-1122, 2015.

[21] F.-y. Mao, H. Kong, Y.-l. Zhao et al., "Increased tumorinfiltrating CD45RA-CCR7- regulatory T-cell subset with immunosuppressive properties foster gastric cancer progress," Cell Death \& Disease, vol. 8, no. 8, article e3002, 2017.

[22] M. Gao, X. Li, L. He et al., "Diammonium glycyrrhizinate mitigates liver injury via inhibiting proliferation of NKT cells and promoting proliferation of Tregs," Drug Design, Development and Therapy, vol. 13, pp. 3579-3589, 2019.

[23] C. Hu, S. Tian, L. Lin, J. Zhang, and H. Ding, "Prognostic and clinicopathological significance of PD-L1 and tumor infiltrating lymphocytes in hypopharyngeal squamous cell carcinoma," Oral Oncology, vol. 102, article 104560, 2020.

[24] F. Li, Y. Sun, J. Huang, W. Xu, J. Liu, and Z. Yuan, "CD4/CD8 $+\mathrm{T}$ cells, DC subsets, Foxp3, and IDO expression are predictive indictors of gastric cancer prognosis," Cancer Medicine, vol. 8, no. 17, pp. 7330-7344, 2019.

[25] E. Peranzoni, V. Ingangi, E. Masetto, L. Pinton, and I. Marigo, "Myeloid cells as clinical biomarkers for immune checkpoint blockade," Frontiers in Immunology, vol. 11, article 1590, 2020.

[26] K. Harada, X. Dong, J. S. Estrella et al., “Tumor-associated macrophage infiltration is highly associated with PD-L1 expression in gastric adenocarcinoma," Gastric Cancer, vol. 21, no. 1, pp. 31-40, 2018.

[27] R. Noy and J. W. Pollard, "Tumor-associated macrophages: from mechanisms to therapy," Immunity, vol. 41, no. 1 , pp. 49-61, 2014.

[28] E. Zhang, F. Dai, Y. Mao et al., "Differences of the immune cell landscape between normal and tumor tissue in human prostate," Clinical \& Translational Oncology, vol. 22, no. 3, pp. 344-350, 2020.

[29] A. Rojas, P. Araya, I. Gonzalez, and E. Morales, "Gastric tumor microenvironment," Advances in Experimental Medicine and Biology, vol. 1226, pp. 23-35, 2020.

[30] G. Sammarco, G. Varricchi, V. Ferraro et al., "Mast cells, angiogenesis and lymphangiogenesis in human gastric cancer," International Journal of Molecular Sciences, vol. 20, no. 9, p. 2106, 2019.

[31] H. Zhang and S. Zhang, "The expression of Foxp3 and TLR4 in cervical cancer: association with immune escape and clinical pathology," Archives of Gynecology and Obstetrics, vol. 295, no. 3, pp. 705-712, 2017.

[32] X. Li, K. Ma, S. Song et al., "Tight correlation between FoxM1 and FoxP3+ Tregs in gastric cancer and their clinical significance," Clinical and Experimental Medicine, vol. 18, no. 3, pp. 413-420, 2018.

[33] Y. Guo, X. Liu, D. Xu et al., "Role of LATS1/2 in prognosis of advanced gastric cancer and its relationship with the tumor immune microenvironment," Frontiers in Oncology, vol. 10, p. $1406,2020$.

[34] J. Borst, J. Hendriks, and Y. Xiao, "CD27 and CD70 in T cell and B cell activation," Current Opinion in Immunology, vol. 17, no. 3, pp. 275-281, 2005.

[35] R. Fristedt, D. Borg, C. Hedner et al., "Prognostic impact of tumour-associated B cells and plasma cells in oesophageal and gastric adenocarcinoma," J Gastrointest Oncol, vol. 7, no. 6, pp. 848-859, 2016.

[36] A. Hennequin, V. Derangère, R. Boidot et al., "Tumor infiltration by Tbet+ effector T cells and CD20+ B cells is associated with survival in gastric cancer patients," Oncoimmunology, vol. 5, no. 2, article e1054598, 2015.

[37] N. C. Sabins, O. Chornoguz, K. Leander et al., “TIM-3 engagement promotes effector memory $\mathrm{T}$ cell differentiation of human antigen-specific CD8 T cells by activating mTORC1," Journal of Immunology, vol. 199, no. 12, pp. 4091-4102, 2017.

[38] Y. Wei, Z. Hu, W. Gu et al., "CD117+CD44+ stem T cells develop in the thymus and potently suppress T-cell proliferation by modulating the CTLA-4 pathway," Stem Cell Research \& Therapy, vol. 8, no. 1, p. 56, 2017.

[39] S. Qin, B. Dong, M. Yi, Q. Chu, and K. Wu, "Prognostic values of TIM-3 expression in patients with solid tumors: a metaanalysis and database evaluation," Frontiers in Oncology, vol. 10, p. 1288, 2020.

[40] X. Liu, Y. Cao, R. Li et al., "Poor clinical outcomes of intratumoral dendritic cell-specific intercellular adhesion molecule 3-grabbing non-integrin-positive macrophages associated with immune evasion in gastric cancer," European Journal of Cancer, vol. 128, pp. 27-37, 2020.

[41] D. Xu, E. Zhao, C. Zhu et al., "TIGIT and PD-1 may serve as potential prognostic biomarkers for gastric cancer," Immunobiology, vol. 225, no. 3, article 151915, 2020.

[42] M. Zhang, G. Hutter, S. A. Kahn et al., “Anti-CD47 treatment stimulates phagocytosis of glioblastoma by M1 and M2 polarized macrophages and promotes M1 polarized macrophages in vivo," PLoS One, vol. 11, no. 4, article e0153550, 2016.

[43] T. A. W. Holderried, L. de Vos, E. G. Bawden et al., "Molecular and immune correlates of TIM-3 (HAVCR2) and galectin 9 (LGALS9) mRNA expression and DNA methylation in melanoma," Clinical Epigenetics, vol. 11, no. 1, p. 161, 2019.

[44] F. Schütz, S. Stefanovic, L. Mayer, A. von Au, C. Domschke, and C. Sohn, "PD-1/PD-L1 pathway in breast cancer," Oncol Res Treat, vol. 40, no. 5, pp. 294-297, 2017.

[45] M. L. Burr, C. E. Sparbier, Y. C. Chan et al., "CMTM6 maintains the expression of $\mathrm{PD}-\mathrm{L} 1$ and regulates anti-tumour immunity," Nature, vol. 549, no. 7670, pp. 101-105, 2017.

[46] L. H. Eusebi, A. Telese, G. Marasco, F. Bazzoli, and R. M. Zagari, "Gastric cancer prevention strategies: a global perspective," Journal of Gastroenterology and Hepatology, vol. 35, no. 9, pp. 1495-1502, 2020.

[47] Z. Song, Y. Wu, J. Yang, D. Yang, and X. Fang, "Progress in the treatment of advanced gastric cancer," Tumour Biology, vol. 39, no. 7, article 101042831771462, 2017. 